\title{
10. PLANETARY NEBULAE IN THE CENTRAL REGION OF THE GALAXY
}

\author{
L. Perek \\ Astronomical Institute of the Czechoslovak Academy of Sciences
}

Planetary nebulae are convenient objects for studying the large-scale structure of the Galaxy. Firstly, they are easily recognized up to considerable distances on plates taken through an objective prism, and secondly, methods have been devised by various authors to determine their distances from two observable quantities: angular diameter and surface brightness. The importance of the subsystem of planetary nebulae has been accentuated especially by the discoveries by Minkowski and Haro of large numbers of planetaries in the direction of the galactic centre. The distribution of planetaries on the sphere suggests that they are connected with the galactic nucleus, but no direct determination of their distances, which would either confirm or contradict this statement, is available. The most serious obstacle in studying the subsystem of planetaries is the lack of observing data. The aim of the reported paper (Perek 1963) is to give a tentative outline of the distribution of planetaries in space based on extensive new observing material.

A region of $20^{\circ}$ in galactic latitude and $50^{\circ}$ in galactic longitude was investigated. Angular dimensions were known previously for 21 planetaries and surface brightnesses for an even smaller number out of a total of 315 planetary nebulae in that region. On direct plates taken with the Tonantzintla Schmidt camera, the Mount Palomar Big Schmidt, the 60-inch, and the 100-inch Mount Wilson reflectors, dimensions have been measured of 345 planetaries. Of these 252 fall into the central region, making a total of 262 measured objects.

With a very few exceptions, the plates were taken on the red-sensitive emulsion Kodak 103aE through a red filter. The sensitivity curve had its maximum at $\lambda 6500$ and the half-intensity points at approximately $\lambda 6200$ and $\lambda 6650$. The most important line in this range is $\mathrm{H} \alpha$ and the two lines of NII at $\lambda 6548$ and $\lambda 6584$ contribute to the total observed light.

On the Schmidt camera plates only planetaries showing a disk or ring and an adequate density were measured. The long focus of the Mount Wilson reflectors made it possible to measure diameters down to $4^{\prime \prime}$ and $3^{\prime \prime}$. Seventy-one planetaries failed to show any difference against a stellar image. Only these were classified as "stellar". They are either too small or too distant, or the envelope is too faint to record on the longest exposure used with that particular nebula. The exposure times were chosen, as far as the limited observing time permitted, so as to give images of medium density. An estimate of the surface brightness was made from the exposure time and the density of the image.

The distances were computed according to Kohoutek's modification of Shklovsky's method. Shklovsky's method (1956) leads to

$$
\log r=0 \cdot 79-\log d^{\prime \prime}+0 \cdot 08 H \text {, }
$$


where $r$ is in kpc, $d^{\prime \prime}$ in seconds of arc, and the surface brightness $H$ in magnitudes per circle of $1^{\prime}$ diameter. The numerical constant contains an assumed average value of the mass of a planetary and the zero point of the distance scale based on statistical parallaxes of a number of planetaries.

Kohoutek (1960) noted that individual masses can be taken into account. The radiation is proportional to the mass, therefore it is sufficient to make an assumption concerning the relation between the radiation of the central star and that of the nebula. Observations suggest that the difference between bolometric magnitudes of the star and of the nebula can be assumed independent of time during the evolution of the nebula. If further a fixed percentage of the total radiation is observed in a specific spectral range, the mass in the distance formula is replaced by the surface brightness $H$. Kohoutek later $(1961,1962)$ extended his method to planetaries with unobserved central stars and, with lower accuracy, to optically thick nebulae. His formula gives

$$
\log r=0 \cdot 41-\log d^{\prime \prime}+0 \cdot 131 H,
$$

where the zero point was determined so as to make the two formulae coincide for the most frequent value of $H=7 \cdot 5$ mag.

Interstellar absorption affects only the surface brightness but it must be taken into account for low latitudes and large distances. The data by Johnson et al. (1961) on the distances of open clusters yield a value of the absorption coefficient valid in the solar neighbourhood, of $1 \mathrm{mag} / \mathrm{kpc}$ at $\mathrm{H} \alpha$. Its variation towards the centre was estimated from the measurements by Vetešník (1962) in the Andromeda nebula. The assumed value at the centre of the Galaxy was $3.5 \mathrm{mag} / \mathrm{kpc}$. From these data a three-dimensional model of galactic absorption was constructed and the distances corrected accordingly. The final formula for the distance was

$$
\log r=0 \cdot 41-\log d^{\prime \prime}+0 \cdot 131(H-A),
$$

where the absorption is a function of direction and distance.

Distances and absorption were computed, besides the 183 planetaries in the central region, for 215. planetaries outside the region. For about 70 of these only diameters and surface brightnesses in photographic light were available.

The distribution of linear diameters is closely related to the distribution of surface brightnesses. The distance formula yields for the linear diameter in astronomical units

$$
\log d=3 \cdot 41+0 \cdot 131(H-A) .
$$

The distribution of planetaries in the central region as well as that of all planetaries with available data shows the maximum frequency at about 25,000 a.u. corresponding to the maximum frequency of $H-A$ between 7 and 8 mag.

An attempt to investigate the distribution of planetaries in space can be made on the basis of the known distances. If the majority of planetaries were situated in the central bulge, as is suggested by the apparent distribution on the sphere, a crowding at a specific distance should be expected. This distance need not necessarily coincide with the distance of the galactic centre since the present zero point of the distance scale may be erroneous. It may be mentioned that the absorption does not essentially change the relative distribution of planetaries. Planetaries are observed 
mostly above the dense parts of the absorbing layer and a shift of a planetary along the radius vector does not much change the absorption at distances beyond $3 \mathrm{kpc}$.

No conspicuous crowding of planetaries is observed and no change of the distance scale would bring them into a cluster around the galactic centre. Planetaries are found at all distances between the Sun and the centre in numbers which do not suggest any major fluctuations of the density. The numbers of planetaries are small near the Sun because of the limitation of the region and they thin out at large distances where the observations become increasingly difficult. There are no planetaries beyond $3 \mathrm{kpc}$ at longitudes between 335 and $350^{\circ}$ because the measurements of diameters are much more incomplete to the south of declination $-35^{\circ}$ than to the north of that limit. At longitudes between 10 and $25^{\circ}$ planetaries are found in much smaller numbers than in the central strip between $l \mathrm{II}=350$ and $10^{\circ}$. No explanation of this puzzling feature is offered, especially as the radio observations do not reveal any abnormal hydrogen densities in that direction.

The absorption in the galactic plane becomes so high beyond $3 \mathrm{kpc}$ that the apparent distribution of planetaries is split into two clouds to the north and to the south of the galactic equator. In the space distribution in a meridian section of the Galaxy a rift appears along the galactic plane.

If all planetaries with available data are plotted they show a more or less uniform scatter in the galactic plane with a preference for the direction, but not for the distance, of the centre. The scatter in the $z$-coordinate is almost constant up to 2 or $3 \mathrm{kpc}$ from the Sun and it increases towards the centre.

The present observations do not suggest any definite change of the adopted distance scale. The spiral structure is totally absent and the most distant planetaries outline possibly the central bulge. These facts confirm that planetary nebulae belong to the disk population of the Galaxy.

More detailed measurements and, particularly, observations of planetaries deep in the southern hemisphere are needed before definite conclusions from the space distributions can be drawn.

\section{References}

Johnson, H. L., Hoag, A. A., Iriarte, B., Mitcheld, R. I., and HallaM, K. L. (1961).-Lowell Obs. Bull. 5 : 133-47.

КоночтEк, L. (1960).-BAC 11 : 64-8.

Коночтек, L. (1961).-BAC 12: 213-24.

KoHOUTEK, L. (1962). - BAC 13: 120.

Perek, L. (1963).-BAC 14: 201.

SHKLOVSKY, I. S. (1956).-AJ USSR 33: 222-35.

VETEŠNfK, M. (1962).-BAC 13: 218-23.

\section{Discussion}

Aller: This valuable contribution adds a great deal to our knowledge of the distribution of planetary nebulae. We must point out, however, that the determination of distance remains the stumbling block. Most of us believe that planetaries evolve from dense small objects to highly attenuated ones. In earlier stages of their lives, the observed radius of the luminous disk is simply that of a Strömgren sphere - the outer part of the shell is non-luminous. Later, the 
gas becomes so attenuated that all of it is ionized. Only for these low surface-brightness nebulae is the method so extensively applied by Shklovsky valid, and there only if all nebulae have the same masses - a question which is by no means settled.

Measurements of the radius in seconds of arc and total brightness in, say, $\mathrm{H} \beta$, i.e. $S(\mathrm{H} \beta)$ in erg $\mathrm{cm}^{-2} \mathrm{sec}^{-1}$ (steradian) ${ }^{-1}$ may be made by comparing the nebula with a suitable star. An error in the distance can produce a gross misinterpretation of the data of the spatial distribution of the nebulae.

The emission per unit volume is

$$
E \sim N_{i} N_{\varepsilon} T_{e}^{-3 / 2} \sim N_{i} T_{\varepsilon}^{-3 / 2}\left(\mathrm{erg} \mathrm{cm}^{-3} \mathrm{sec}^{-1}\right)
$$

The surface brightness for a uniform volume is

and the mass of the planetary

$$
S(\mathrm{H} \beta)=\frac{4}{3} \pi r^{3} E / 4 \pi r^{2}=\frac{1}{3} E r
$$

$$
M_{p} \cong \frac{4}{3} \pi r^{3} N_{i} M_{H}
$$

so

$$
S(\mathrm{H} \beta) \propto M_{p}^{2} r^{-5} T_{\epsilon^{-3 / 2}} .
$$

If $M_{1}<M_{p}<M_{2}$ all you can say by comparing $\mathrm{d}^{\prime \prime}$ and $r(\mathrm{~cm})$ is that the distance, $R$, lies between limits $R_{1}<R<R_{2}$. So really all this method is useful for is setting distance limits on a given nebula, as Minkowski and Aller used it for getting the limits on distance of the Owl nebula.

Perek: The surface brightnesses of almost all planetaries in our material are fainter than 5 mag per circle of $\mathbf{l}^{\prime}$ in diameter, which is mentioned by Shklovsky as the limit of optically thin planetaries. About 80 stellar planetaries were not included in the discussion. Moreover, the limitation to optically thin planetaries is less strict for Kohoutek's modification of the method. It was not assumed that planetaries have the same masses. The assumptions which were made have been mentioned in the text.

The nature of the investigation is, of course, quite preliminary and it would be too much to expect reliable individual distances from it.

Bok: What are the values of your average and maximum absorption corrections?

Perek: The maximum absorption correction is $4 \cdot 7$ mag. The average correction is between 1 and 2 mag.

Oort: I think the fact that in galactic longitudes there is so strong a concentration of planetaries near the direction of the centre shows forcibly that the bulk of the objects observed in that direction are actually near the galactic centre. It the plots of space coordinates do not show a concentration at the distance of the centre, this indicates that the estimated distances are in error.

Perek: I too expected that the majority of planetaries observed in the direction of the centre would be at its distance. Since no concentration of planetaries appeared at any distance, it is not evident from the available material in which direction the distance scale should be changed.

Lindblad: Are not the radial velocities known for a great number of planetaries, which might check the distance scale to some extent?

Perek: A large number of radial velocities were measured by Minkowski. The planetaries are, however, so close to the direction of the galactic centre that the Camm function has very small weight.

Oort: The use of radial velocities for obtaining distances is rendered difficult because of the large random motions, especially in the region of the centre.

Blaauw: In order to obtain a concentration in the centre region, a change in the distance scale would not do; the principle of the method would have to be modified so that the ratios of the distances will be revised.

Perek: I agree.

Carter: Does the distribution in space change if one takes the angular size as a rough scale? 
Perek: No, it does not change. This point is illustrated by the slide showing the distribution of angular diameters.

Westerlund: Henize and I have photographed with the 74-inch telescope at Mount Stromlo 165 southern objects out of a list of 283 , half of which are new. It will be followed up, we hope, with $\mathrm{H} \beta$ photometry and spectroscopy. We hope to publish identification charts (in $\mathrm{H} \alpha$ ) of all objects. I think the compilation of a general catalogue a very good idea.

Thackeray: Is it possible that there is a very strong observational selection whereby one loses many planetaries at distances greater than, say, $8 \mathrm{kpc}$ ?

Perek: If the distance scale is correct, we observe only a few planetaries at a distance greater than $8 \mathrm{kpc}$. A search for still fainter and smaller planetaries, carried out with a longfocus Schmidt camera and in excellent seeing, would be necessary to settle the question.

\title{
11. SPECTROPHOTOMETRY OF 14 SOUTHERN PLANETARY NEBULAE
}

\author{
L. H. ALLER* \\ University of California, Los Angeles
}

D. J. FaULKNer

Mount Stromlo Observatory

The theory of radiative and collisional processes in gaseous nebulae makes possible precise predictions of line and continuum intensities as functions of density, electron temperature, and degree of ionization of the emitting gases. It predicts, for example, the relative intensities of successive members of the Balmer series (Balmer decrement), and of corresponding transitions in the Balmer and Paschen series. Hence accurate line intensity measurements are not only of astrophysical interest; they also serve to assess the amount of space absorption.

Spectrophotometry may be done either photographically or photoelectrically. As far as isolated strong lines are concerned there is little question that the photoelectric technique is the more advantageous. For weaker lines, or for certain close pairs such as 3968 [NeIII], $3970 \mathrm{H}$, or 3726, 3729 [OII], high resolution, such as can be attained only by photographic techniques, is necessary.

In 1956 at the Mount Wilson Observatory, Liller and Aller used a photoelectric spectrophotometer designed and built by the former (Liller 1957) at the University of Michigan, to measure the relative intensities of stronger lines in northern planetary nebulae. The present program constitutes an extension of this work to the southern hemisphere, except that we were unable to secure observations in the red and infrared.

This scanner is supplied with slots and diaphragms of different sizes to accommodate objects of various angular diameters and to obtain spectral resolutions ranging from better than $24 \AA$ up to $100 \AA$. Scanning speeds ranging from $30 \AA / \mathrm{min}$ to $270 \AA / \mathrm{min}$ were available. Since the instrument was originally designed for an $f / 5$ system and had to be used on telescopes with aperture ratios of $f / 12$ and $f / 18$, it was necessary to add auxiliary optics in the entrance beam.

* Senior National Science Foundation post-doctoral Fellow at Australian National University, 1960-1961. 